\title{
Induced movement as nonveridical resolution of displacement ambiguity: Effect of enclosure and number of field elements
}

\author{
R. H. DAY, JANE MILLAR, and R. G. DICKINSON \\ Monash University, Victoria, 3168, Australia
}

\begin{abstract}
Four experiments on induced movement and induced stationariness are described. Experiments 1 and 2 showed that mere enclosure of a stationary spot in a moving frame does not necessarily result in induced movement. Nor does enclosure of a moving spot in a stationary frame necessarily result in perceived real movement of the spot. Duncker's principles of enclosure is thus called into question. Two further experiments showed that both induced and perceived real movement of a spot are much more frequent when the frame is replaced by either two or more similar spots which enclose or flank the target spot. It can be concluded that the principle of enclosure obtains when the reference field consists of more than one element which move or remain stationary together. When such a field moves, it is the single, enclosed element which appears to move while the field itself appears stationary.
\end{abstract}

It was shown earlier (Day, 1978) that when one of two small spots of light moves slowly in an otherwise dark field, either of the spots is perceived to move. Which of the two appears to move is a matter of chance. Occasionally it's the moving spot that appears to move, and occasionally it's the stationary spot. This state of affairs is changed by the addition to the display of a surrounding frame. When the two spots are enclosed in a stationary frame, perception is veridical; the moving spot is almost always seen to move and the stationary spot to remain stationary. However, when the frame moves in the same direction and at the same speed as the moving spot perception is nonveridical; the stationary element is usually seen to move in the opposite direction to the frame (induced movement) and the moving element to remain stationary (induced stationariness). These states of affairs obtain when the speed of the moving spot or frame does not exceed the subject-relative threshold, the threshold for movement of an object relative to the observer in an otherwise completely dark field (Kinchla, 1971). ${ }^{1}$ When the speed of the moving elements of the display progressively exceeds this threshold, veridical perception is increasingly common.

These outcomes confirmed that for speeds and intervals below the subject-relative threshold, displacement of one spot relative to another in a dark field is ambiguous (Day, 1978; Kinchla, 1971; Wallach, 1959). They also indicated that the frame or field serves as a perceptually stationary reference regardless of whether it is actually stationary or moving. In the case of the more conventional display, consist-

The assistance of the Australian Research Grants Committee is gratefuly acknowledged. ing simply of a single spot and a surrounding frame, it was concluded that the ambiguous displacement of one relative to the other would be resolved veridically or nonveridically on the same basis. As will be clear below, this conclusion was not justified.

The results from this earlier investigation left unanswered the key question of what characterizes a frame of reference or reference field which is perceptually stationary and in terms of which the ambiguous displacement is resolved as spot movement. The four experiments reported here were concerned with this issue. In Experiments 1 and 2, the role of enclosure of a spot by a field was investigated. Since enclosure by itself was unexpectedly found not to be a determinant of either veridical or nonveridical resolution, and since in the earlier experiments two moving elements, a spot and the frame, were involved, Experiments 3 and 4 were concerned with the joint effects of enclosure and the number of elements constituting the reference field. In Experiment 3, the field consisted of two spots which always moved or remained stationary together; in Experiment 4 the field consisted of six such spots.

\section{EXPERIMENT 1}

In discussing his first series of experiments Duncker (1929) stated: "Thus we have encountered two determinants of perceived motion: (1) other things being equal, there is a greater tendency for motion to appear in the fixated object than in the non-fixated one; (2) the same as regards the enclosed rather than the enclosing object. Stated in general terms we may say that when an object shifts relative to its frame of reference, phenomenal motion of that object occurs" (pp. 163-164). 
In two experiments designed to evaluate the role of the first of these determinants, Mack, Fisher, and Fendrich (1975) have shown that the nonfixated element in a two-point display does not serve as the frame of reference for perceived movement of the fixated element. The second determinant, that of enclosure, has not been systematically investigated, although it is widely assumed that it is the most salient single determinant of induced movement (Kaufman, 1974; Rock, 1975). The purpose of the first experiment was to establish whether enclosure of a point by a frame was a determinant of induced movement when the point was stationary and of perceived real movement when it moved. A moving or stationary spot was located inside and outside a rectangular frame. The subject's task was simply to indicate which moved during a 2-sec exposure, spot or frame.

\section{Method}

Subjects. There were 12 paid subjects, 7 women and 5 men, all of whom were undergraduates, graduate students, or technical assistants.

Apparatus. The apparatus has already been described (Day, 1978; Day \& Dickinson, 1977). Its essential features were two clear Plexiglas slides, back-illuminated by diffused light. One of these was frontoparallel and viewed through a half-silvered mirror tilted forward at $45^{\circ}$, and the other was horizontal and reflected from the front surface of the mirror. Cutouts which could be closely fixed to the surfaces of the slides produced the stationary and moving elements of the stimulus pattern. Both elements appeared frontoparallel and in the same plane in an otherwise dark, featureless field. The slides could be moved laterally to the right or left by means of a motor operating through a rack and pinion system. The viewing distance, maintained by the use of a chinrest, was $287 \mathrm{~cm}$, at which $50 \mathrm{~mm}$ subtends a visual angle of $1^{\circ}$.

Stimulus patterns. The relative dimensions of the spot and frame and their four arrangements are shown in Figures 1A-1D. Their dimensions were such that the total lighted area of each was $1,000 \mathrm{~mm}^{2}$. In terms of visual subtense, the frame was $.3^{\circ}$ horizontally and $.2^{\circ}$ vertically in outside measurement, and the frame $.04^{\circ}$ between inside and outside edges. The spot was $.072^{\circ}$ in diameter. The luminance of both elements was $.5 \mathrm{~cd} / \mathrm{m}^{2}$. The

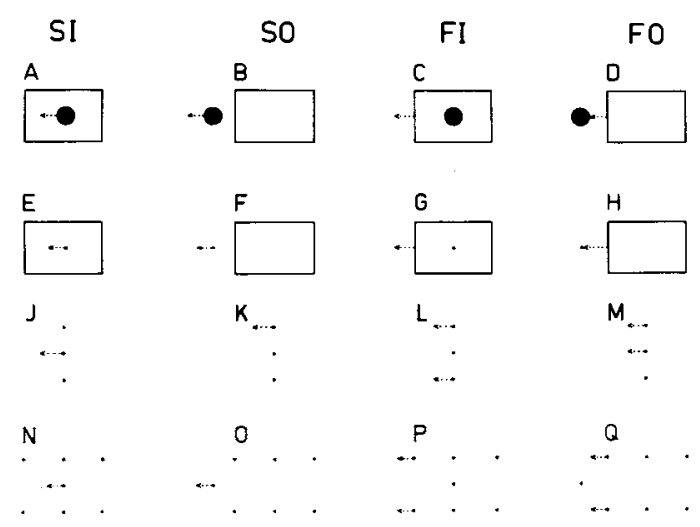

Figure 1. The stimulus patterns used in the three experiments. The arrows indicate the moving elements.
Table 1

Means and Standard Deviations of the Frequencies With Which the Single Spot was Perceived to Move in the Four Experiments

\begin{tabular}{|c|c|c|c|c|c|c|c|c|}
\hline \multirow{3}{*}{$\begin{array}{l}\text { Exper } \\
\text { iment }\end{array}$} & \multicolumn{8}{|c|}{ Stimulus Conditions } \\
\hline & \multicolumn{2}{|c|}{ SI } & \multicolumn{2}{|c|}{ So } & \multicolumn{2}{|c|}{ FI } & \multicolumn{2}{|c|}{ FO } \\
\hline & Mean & SD & Mean & SD & Mean & SD & Mean & SD \\
\hline $\begin{array}{l}1 \\
2 \\
3 \\
4\end{array}$ & $\begin{array}{l}5.00 \\
6.51 \\
6.08 \\
7.58\end{array}$ & $\begin{array}{l}1.65 \\
1.68 \\
1.51 \\
0.67\end{array}$ & $\begin{array}{l}4.58 \\
5.63 \\
4.33 \\
5.42\end{array}$ & $\begin{array}{l}2.15 \\
2.15 \\
1.23 \\
1.83\end{array}$ & $\begin{array}{l}4.92 \\
5.02 \\
6.25 \\
6.58\end{array}$ & $\begin{array}{l}1.98 \\
2.26 \\
1.48 \\
1.73\end{array}$ & $\begin{array}{l}3.58 \\
5.34 \\
4.83 \\
5.67\end{array}$ & $\begin{array}{l}2.27 \\
1.97 \\
1.40 \\
1.83\end{array}$ \\
\hline
\end{tabular}

Note-SI and SO refer respectively to real movement of the spot when inside, i.e., enclosed by, and outside, i.e., not enclosed by, the stationary frame or spots. FI and FO refer respectively to real movement of the frame with the stationary spot inside and outside it. The maximum score was 8.

speed of the moving element during the 2 -sec exposure was $.1 \% \mathrm{sec}$, well below the reported subject-relative movement threshold for this interval (Brown \& Conklin, 1954; Mack, Fisher, \& Fendrich, 1975).

Procedure. The subject's task throughout was simply to report whether the spot or the frame moved. There were 32 trials, 8 for each of the four conditions designated as follows: SI, spot moving inside stationary frame (Figure 1A); SO, spot moving outside stationary frame (Figure 1B); FI, spot stationary inside moving frame (Figure 1C); FO, spot stationary outside moving frame (Figure 1D). For half the trials in each condition, the spot or the frame moved to the right and for the other half to the left. The starting positions were always those shown in Figure 1A-1D, with the spot either centered in the frame or to the left of it at the same distance from the frame edge $(75 \mathrm{~mm})$. The order of presentation of the 32 trials was separately randomized for each subject. Recorded instructions which stressed the importance of a quick impressionistic response were played at the beginning of each session and questions encouraged and answered. At the beginning of a trial, the experimenter signaled "ready," after which the subject opened his eyes, indicated as quickly as possible whether the spot or the frame appeared to be moving, and closed his eyes again and kept them closed until the next trial. Since it has been shown (Mack, Fisher, \& Fendrich, 1975) that which of two elements appears to move and which to remain stationary is not dependent on which of the two is fixated, the subjects were not specifically instructed to look at the spot or at the frame. The intertrial interval was about 10-15 sec. The score for each condition was the number of judgments of spot movement with a maximum possible score of 8. Thus, if the score was 5 , it indicated that for five trials the spot was reported as moving and for three, the frame. Depending on the condition, the score indicated either perceived real movement of the spot when it was actually moving or induced movement when it was stationary. In the former case, the frame was stationary and in the latter it was moving.

\section{Results}

The mean scores for perceived spot movement for the four conditions are shown with their standard deviations in Table 1. Inspection of these means does not suggest a strong effect of enclosure on the frequency with which either real or induced movement was perceived or, indeed, even a tendency for the enclosed spot rather than the frame to be perceived as moving. Both these initial impressions were confirmed by statistical analysis. The effect of enclosure of the spot in the frame and of spot and frame 
movement were examined in a 2 by 2 analysis of variallce with repeated measures on both factors. Neilher the position of the spot relative to the frame nor movement of the spot or frame significantly affected perceived movement of the spot $[F(1,11)=$ $1.55, \mathrm{p}>.05 ; \mathrm{F}(1,11)=3.30, \mathrm{p}>.05$, respectively]. The interaction between these variables also failed to achieve significance $[F(1,11)=1.66, p>.05]$.

The method used to test whether the frequency of perceived spot movement exceeded chance in each condition was to compare the obtained mean score with the chance score of 4 using a t test with $\mu=4$. However, since for the four experiments there was a total of 16 such comparisons, 4 for each experiment, the $\alpha$ level was set at .005 , for which the probability of rejecting the null hypothesis when it was true was approximately .04 (Winer, 1962, p. 199).

The outcomes of the four $t$ tests indicated that there was no significant difference between the obtained and chance mean scores for perceived spot movement $\left[\mathrm{t}_{\mathrm{SI}}(11)=2.10, \mathrm{p}>.025 ; \mathrm{t}_{\mathrm{FI}}(11)=1.61\right.$, $\mathrm{p}>.05 ; \mathrm{t}_{\mathrm{SO}}(11)=.94, \mathrm{p}>.05 ; \mathrm{t}_{\mathrm{FO}}(11)=.63$, $\mathrm{p}>$.05].

In summary, enclosure of a moving or stationary spot in a stationary or moving frame did not significantly affect the frequency with which either real or induced movement of the spot was perceived compared with nonenclosure. Furthermore, there was no difference between perceived real movement of the spot when the frame was stationary and its induced movement when the frame was moved. Finally, contrary to expectation, the frequency with which the spot was perceived to move did not exceed chance under any of the four conditions; perceived movement of the frame was as probable as that of the spot.

\section{EXPERIMENT 2}

In the first experiment, the size of the enclosed spot relative to the rectangular area within the frame was considerably greater than usual in experiments on induced movement (Brosgole, 1968; Gogel, 1977; Wallach, 1959). It will be recalled that the relative sizes of spot and frame were based on the decision to make the two elements equal in area. It is conceivable that the effect of enclosure is manifested only when the spot is much smaller relative to the enclosed area of the frame than obtained in Experiment 1 . In the second experiment, therefore, the spot was considerably smaller. In all other respects, the stimulus pattern was the same.

\section{Method}

Subjects. There were 12 new subjects, 6 women and 6 men, all of whom were undergraduates, graduate students, or technical assistants.
Apparatus. The apparatus was the same as in Experiment 1.

Stimulus patterns. The relative dimensions and the four arrangements of the spot and frame are shown in Figures 1E-1H. The spot, which was markedly smaller than in Experiment 1, was $.01^{\circ}$ in diameter and $6 \mathrm{~mm}^{2}$ in area. The dimensions of the frame and the luminance and speed of the spot and frame were identical to those of the first experiment.

Procedure. The procedure, including instructions and scoring, was the same as in Experiment 1.

\section{Results}

The mean scores for perceived spot movement for the four conditions are shown with their standard deviations in Table 1. While inspection of the means suggests that they are generally greater than in Experiment 1 , it again does not suggest a difference between the conditions of enclosure and nonenclosure. An analysis of variance similar to that carried out earlier showed, in fact, that the difference in mean perceived spot movement between the enclosure and nonenclosure conditions was not significant $[\mathrm{F}(1,11)=.57$, $p>.05]$, but that between movement of the spot and frame, i.e., between perceived real movement and induced movement of the spot, was $[F(1,11)=13.36$, $p<.01]$. The interaction between the two variables did not achieve significance $[F(1,11)=1.42, p>.05]$. Separate $t$ tests showed that perceived spot movement exceeded the chance score of 4 only in Condition SI $\left[\mathrm{t}_{\mathrm{SI}}(11=5.15, \mathrm{p}<.005]\right.$. For the other three conditions, the difference between the obtained means and chance was not significant in terms of the .005 criterion $\left[\mathrm{t}_{\mathrm{FI}}(11)=1.54, \mathrm{p}>.05 ; \mathrm{t}_{\mathrm{SO}}(11)=2.55, \mathrm{p}>.01\right.$; $\left.\mathrm{t}_{\mathrm{FO}}(11)=2.35, \mathrm{p}>.025\right]$.

These results show that with a spot considerably smaller in area than the frame border, enclosure of the spot within the frame did not result in greater frequencies of either perceived real movement or induced movement of the spot compared with nonenclosure. However, perceived real movement of the spot occurred more frequently than did induced movement. In only one condition, spot moving in the stationary frame, did perceived spot movement exceed chance.

\section{EXPERIMENT 3}

In the experiments reported earlier (Day, 1978), subjects undertook a different task to that of Experiments 1 and 2. They reported which of two spots in a frame moved. When the frame and one spot moved, induced movement of the other, stationary spot occurred with almost maximum frequency, and when the frame and one spot were stationary, perceived real movement of the other moving spot was nearly maximal. Leaving aside the obvious differences in the subject's tasks, the two groups of experiments differed in respect to the relative number of moving or stationary stimulus elements in the 
field. In the earlier group, either two elements moved while one, a spot, was stationary, or vice versa. In the two experiments so far reported here, only one of the two elements, the spot or frame, moved while the other was stationary. With this difference in mind, it seemed possible that ind: id and perceived real movement of a single element occurs when more than one element in the field moves or remains stationary. This possibility was tested in the third experiment. Instead of a spot and frame, a pattern consisting simply of three identical spots was used. While one of these remained stationary or moved, the other two moved or were stationary, respectively. That is, the field or frame of reference consisted of a pair of spots, each identical to the target spot.

\section{Method}

Subjects. There were again 12 subjects, 7 women and 5 men, trom the same sources as earlier. Two had participated in one of the two earlier experiments but were unfamiliar with the purpose of the experiment.

Apparatus. The apparatus was the same as for Experiments 1 and 2.

Stimulus patterns. The stimulus patterns, shown in Figures $1 \mathrm{~J}$ $1 \mathrm{M}$, each consisted of three identical spots arranged so that one was stationary while a pair moved, or vice versa. Under both these conditions, one spot was either enclosed between the pair or located above or below the remaining pair. ${ }^{2}$ Each spot subtended a visual angle of $.072^{\circ}$, as before. The outer spots corresponded with the positions of the horizontal borders of the frame in the earlier experiments and were $.18^{\circ}$ between centers. The center spot was halfway between the outer two. The luminance and speed of movement was the same as before. For convenience, the four conditions are designated similarly to the earlier experiments: SI, center spot moving between the stationary pair; SO, single spot moving above or below a stationary pair; FI, the outer pair moving with center spot stationary; FO, a pair of spots moving with stationary spot above or below.

Procedure. The subject's task was to indicate whether one spot or a pair of spots moved during the 2 -sec period by saying "top," "middle," or "bottom" when one appeared to move and "top and middle," "middle and bottom," or "top and bottom" when a pair appeared to do so. Appropriate recorded instructions were played at the beginning of each session, and questions were encouraged and answered. The procedure was the same as that described for the two earlier experiments, except that a pair of spots was substituted for the frame.

\section{Results}

The mean scores for perceived movement of the single spot for the four conditions are shown in Table 1 . It can be noted that, for the two conditions in which the spot was enclosed between the pair of spots, the frequency of both perceived real movement and induced movement was greater than when it was not so positioned, but that there were no marked differences between the frequency of the two types of movement. A 2 by 2 analysis of variance similar to that carried out in the two earlier experiments showed that the effect of enclosure was significant $[F(1,11)=9.09, p<.05]$, but that the effects of movement of the spot or pair of spots was not $[F(1,11)=1.44, p>.05]$. The interaction between these two variables also failed to achieve significance $[F(1,11)=.39, p>.05]$. Separate $t$ tests to compare the scores with the chance score of 4 were again carried out. These showed that for the two conditions in which the spot was enclosed, the frequency of its perceived movement significantly exceeded chance $\left[\mathrm{t}_{\mathrm{SI}}(11)=4.80, \mathrm{p}<.005 ; \mathrm{t}_{\mathrm{FI}}(11)=\right.$ $5.25, \mathrm{p}<.005$ ], but for the two in which the spot was not enclosed it did not [ $\mathrm{t}_{\mathrm{SO}}(11)=.94, \mathrm{p}>.05$; $\left.\mathrm{t}_{\mathrm{FO}}(11)=2.06, \mathrm{p}>.05\right]$.

\section{EXPERIMENT 4}

The results of Experiment 3, along with those of the earlier series (Day, 1978) indicate that enclosure of an element in a field consisting of more than one element results in a significantly greater frequency of perceived real movement and induced movement of the former than does nonenclosure. In view of this outcome, it is reasonable to suppose that the frequency of perceived movement of an enclosed spot would be even greater if the field elements were more numerous than in Experiment 3. In the final experiment, therefore, the single spot was either enclosed or unenclosed by a field of six spots forming two rows of three. In the interest of comparing the results with those of Experiments 1 and 2, in which a frame was involved, the two rows of spots corresponded with the upper and lower borders of the frame used in Experiments 2 and 3.

\section{Method}

Subjects. There were 12 new subjects, 6 women and 6 men, drawn from the same sources as before.

Apparatus. The apparatus was the same as for the three earlier experiments.

Stimulus patterns. The four stimulus patterns are shown in Figures 1N-1Q. The upper and lower rows of equidistant spots corresponded with upper and lower borders of the rectangular frame in Experiments 1 and 2, with the outer ones corresponding with its corners. The spots were the same size as before. Thus, the pattern, including movement of the single spot and the field of spots, was the same as in Experiments 1 and 2, excepting that the frame was replaced by two parallel rows of spots.

Procedure. The procedure was the same as in the earlier experiments.

\section{Results}

The mean frequencies of perceived spot movement under the four conditions are shown with their standard deviations in Table 1. It can be seen that, for the SI and FI conditions, the frequency of perceived movement of the spot was greater than for the unenclosed conditions. It is worth noting also that the score for SI was marginally greater than in Experiment 3 but that for FI was about the same. Furthermore, the scores for the two unenclosed conditions, SO and FO, were slightly greater than in Experiment 3. 
A 2 by 2 analysis of variance similar to those carried out in the earlier experiments showed that the effect of enclosure was significant $[F(1,11)=28.52$, $\mathrm{p}<.011$, but that for single or multiple spot movement was not $[F(1,11)=4.69, p>.05]$. The difference between each of the four means and the chance score of 4 was again tested by means of separate $t$ tests. The outcomes showed that for the two conditions in which the spot was enclosed between the rows the means exceeded chance $\left[\operatorname{tsI}_{\mathrm{SI}}(11)=18.57\right.$, $\left.\mathrm{p}<.005 ; \mathrm{t}_{\mathrm{FI}}(11)=5.17, \mathrm{p}<.005\right]$, while for the two in which the spot was located to the left of the two rows only that in which the single spot actually moved achieved significance $\left[\mathrm{t}_{\mathrm{SO}}(11)=3.16, \mathrm{p}<\right.$ .005 ]. For Condition FO, i.e., induced movement of the spot when not enclosed by the rows, the score was not significantly different from chance $\left[\mathrm{t}_{\mathrm{FO}}(11)\right.$ $=2.68, \mathrm{p}<.025]$.

Thus, the results of the final experiment, in addition to confirming those of Experiment 3, suggest that increasing the number of field elements beyond two does slightly increase the frequency of perceived real movement for both the enclosure and nonenclosure conditions. However, the addition and arrangement of field elements did not appear to affect the frequency of induced movement for either condition.

\section{DISCUSSION}

The results from the four experiments can be summarized as follows. For displays consisting of a single spot and a frame, the frequency of perceived real movement and induced movement of the spot was the same whether it was enclosed by the frame or not. Apart from one condition of the eight in the first two experiments, the frequency of perceived spot movement was no greater than chance. For displays consisting of a single spot and either a pair or two rows of similar spots, the frequency of both perceived real movement and induced movement of the single spot was greater when it was flanked by or enclosed between the others than when it was not so.

Two main conclusions can be drawn from these results. First, enclosure of a moving or stationary element in a frame is not by itself sufficient for more than chance occurrence of either perceived real movement or induced movement of the element. Second, if the single frame is replaced by two or more separate elements which together remain stationary or move, then both real and induced movement of a single, similar element is more frequent when it falls between them than when it does not. Enclosure of one element by another, as in the spot-frame arrangement, is not alone sufficient to resolve the perceptually ambiguous displacement of one element from another. However, if the surrounding field consists of two or more elements, then the single, surrounded element appears to move more frequently than the field elements.

The finding that, apart from one condition in the first two experiments, perceived spot movement did not depart significantly from chance was unexpected. It implies that perceived movement of the spot and frame for this stimulus arrangement is equiprobable.

Why, then, has it for so long been accepted that the moving frame serves as a perceptually stationary reference relative to which the stationary point is mostly, or always, perceived to move? The answer to this question probably lies in the methods conventionally used to record and measure induced visual movement. The observer is usually required to cancel or manually to track the perceived movement of the point, never that of the field. The outcome is invariably that induced or real movement of the point is indicated. When, however, the observer is invited to indicate which element appears to move, point or frame, perceived movement of one is as common as that of the other. In support of the generality of this finding, it is worth pointing out that in an earlier series of unpublished experiments in which subjects tracked real and induced movement of the point and the frame, perceived real movement of the frame was as common as that of the point. Induced movement of the frame was less common than that of the point, but nevertheless significant. It seems likely, therefore, that the frequently claimed principle of surroundedness with the point-frame display derives from the confinement of judgments to the point itself. An informal observation involving the stationary moon and a moving cloud-a situation to which reference is frequently made-strongly supports this argument. If, rather than attending to induced movement of the moon, attention is given to the moving cloud, the latter will be clearly seen to move. A number of observers have confirmed this observation by casual inspection. That is, if the field is attended to, it is reported as moving.

It is emphasized that while, in Experiments 3 and 4 , enclosure resulted in significantly greater frequencies of both real and induced movement of the single spot, the frequencies nevertheless were below maximum. That is, occasionally the field spots were perceived to move. In the earlier experiments (Day, 1978), in which an enclosing frame and a spot moved or remained stationary, both induced movement and perceived real movement of the second spot was virtually maximal. It is reasonable to assume that in the earlier experiments the configuration consisting of a frame and a spot constituted a more salient reference field than two or six spots, as in the experiment described here. A series of informal observations designed to compared the frequencies of spot movement with these two field configurations suggests 
that the frequency with the former field are maximal. These observations have yet to be confirmed.

Finally, the greater frequency of perceived real movement in Experiment 2 deserves some comment. This result is difficult to explain. Given that the speed of movement and the exposure interval accorded with those reported to result in subthreshold subjectrelative movement (Brown \& Conklin, 1954; Mack, Fisher, \& Fendrich, 1975), it seems unlikely that displacement of the spot relative to the observer himself was responsible for this result. If this were so, then a higher frequency of perceived real movement would have occurred in Experiment 1 also.

\section{REFERENCES}

Brosgole, L. An analysis of induced motion. Acta Psychologica, $1968,28,1-44$.

Brown, R. H., \& ConkıIN, J. E. The lower threshold of visible movement as a function of exposure-time. American Journal of Psychology, 1954, 67, 104-110.

DAY, R. H. Induced visual movement as nonveridical resolution of displacement ambiguity. Perception \& Psychophysics, 1978, 23, 205-209.

DAY, R. H., \& Dickinson, R. G. Absence of color-selectivity in Duncker-type induced visual movement. Perception \& Psychophysics, 1977, 22, 313-320.

DunCKer, K. Über induzierte Bewegung (Ein Beitrag zur Theorie optisch wahrgenommener Bewegung). Psychologische Forschung, 1929, 12, 180-259.

GOGEL, W. C. Independent motion induction in separated portions of the visual field. Bulletin of the Psychonomic Society, 1977, 10, 408-410.

Kaufman, L. Sight and mind: An introduction to visual perception. New York: Oxford University Press. 1974.
Kinchla, R. A. Visual movement perception: A comparison of absolute and relative movement discrimination. Perception \& Psychophysics, 1971, 9, 165-171.

Mack, A., Fisher, C. B., \& Fendrich, R. A re-examination of two-point induced movement. Perception \& Psychophysics, 1975, 17, 273-276.

Rock, I. An introduction to perception. New York: Macmillan, 1975.

Wallach, H. The perception of motion. Scientific American, 1959, 201, 56-60.

WINER, E. J. Statistical principles in experimental design (2nd ed.). New York: McGraw-Hill, 1962.

\section{NOTES}

1. Since subject-relative movement involves a change of position of the stimulus object in the dark relative to the observer himself, the detectability of movement varies with both stimulus velocity and the duration of exposure. Thus, the change of position can be detected during a short exposure if velocity is high enough but only during a longer interval if it is low. In the experiments described here, exposure duration was always $2 \mathrm{sec}$. Brown and Conklin (1954) and Mack, Fisher, and Fendrich (1975) found that the threshold velocity for this interval was about $13^{\prime} / \mathrm{sec}$.

2 . In the interests of uniformity, the term enclosure is used throughout to refer to complete enclosure of the spot within the unbroken border of a rectangle, as in Experiments 1 and 2, its position between a pair of similar spots in Experiment 3, and its position between two rows of spots in Experiment 4. In the latter arrangement, the term "flanking" rather than "enclosure" is a more apt description.

(Received for publication March 14, 1978; revision accepted October 20, 1978.) 